\title{
Du rang à la ville : le processus d'urbanisation au Québec et en Ontario
}

\author{
Richard S. Alcorn et José E. Igartua
}

Volume 29, numéro 3, décembre 1975

URI : https://id.erudit.org/iderudit/303465ar

DOI : https://doi.org/10.7202/303465ar

Aller au sommaire du numéro

Éditeur(s)

Institut d'histoire de l'Amérique française

ISSN

0035-2357 (imprimé)

1492-1383 (numérique)

Découvrir la revue

Citer cette note

Alcorn, R. S. \& Igartua, J. E. (1975). Du rang à la ville : le processus d'urbanisation au Québec et en Ontario. Revue d'histoire de l'Amérique française, 29(3), 417-420. https://doi.org/10.7202/303465ar d'utilisation que vous pouvez consulter en ligne. 
NOTE DE RECHERCHE

\title{
DU RANG À LA VILLE : LE PROCESSUS D'URBANISATION AU QUÉBEC ET EN ONTARIO
}

\author{
RICHARD S. ALCORN \\ Département d'bistoire \\ University of Western Ontario \\ JOSÉ E. IGARTUA \\ Département d'histoire \\ University of Western Ontario
}

L'histoire urbaine au Canada a marqué, depuis quelques années, des progrès sensibles. Un nombre grandissant d'enquêtes sont en cours sur l'origine et le développement de certaines grandes villes canadiennes, dont Montréal, Québec, Toronto, Hamilton, Halifax. Parallèlement, les milieux ruraux font aussi l'objet d'études systématiques. On pense, par exemple, aux travaux de Cole Harris, Fernand Ouellet et Normand Séguin pour le Québec, et aux recherches du groupe «Peel County» pour l'Ontario. De chaque côté, l'on est fort conscient de la coupure artificiellement pratiquée entre villes et campagnes. Ni la ville ni la campagne n'existent sans rapports l'une avec l'autre, mais le travail monographique doit s'imposer des limites.

Les rapports villes-campagnes intéressent les historiens canadiens depuis longtemps. Tous connaissent les travaux de N.S.B. Gras et la thèse du métropolitanisme de Careless. Mais si les modèles implicites dans l'œuvre de Gras ou celle de Careless peuvent être de quelque secours pour comprendre l'évolution des «grandes» villes, ils sont beaucoup moins utiles à l'étude des zones grises qui marquent le passage du rang à la ville. Ces zones sont-elles urbaines ou rurales? Où tirer la ligne? Dans les recensements canadiens, «urbains» et «ruraux» sont définis par la loi: l'ensemble des villes et des villages constitués, c'est-à-dire un niveau minimal $x$ de popu- 
lation sur une superficie définie, plus ou moins arbitrairement, par les législations provinciales. Les recensements laissent donc pour compte l'univers des comportements, des attitudes et des mentalités. Ils ont pour autre inconvénient de modifier d'une décennie à l'autre, et souvent, dans un même recensement, d'une province à l'autre, la définition d' «urbain». Il est donc malaisé d'utiliser les ventilations publiées dans les recensements pour délimiter le continuum ruralurbain et pour y situer telle ou telle localité. (Voir la critique de ces ventilations publiée en annexe du recensement de 1931, vol. 13: 443-445.)

Comment donc aborder la dynamique du processus d'urbanisation? Il faudrait en premier lieu identifier les sens possibles des termes «urbain» et «rural». Densité de population, bien sûr, mais aussi modes de production et d'échange, et donc comportements, voire attitudes? «Urbain» et «rural» servent-ils à désigner un milieu, un comportement, le jeu du milieu sur le comportement, ou les trois à la fois? Chaque définition devient, de fait, normative; chacune peut constituer un modèle. La vérification empirique de ces modèles nous amène d'abord à étudier le milieu et à tracer les relations d'échange villes-campagnes, c'est-à-dire les relations d'échange entre les localités d'un territoire donné. On pourra ensuite procéder à une schématisation du processus d'urbanisation en tant que transformation du milieu et fixer des repères sur le multiple continuum ruralurbain. Des études plus fouillées sur des localités-types permettront peut-être de déboucher sur les comportements et les attitudes, et ainsi approfondir les concepts d' «urbain» et de «rural» .

C'est là une démarche ambitieuse. Le projet que nous avons entrepris est plus modeste. Il vise pour l'instant à esquisser quelquesunes des relations d'échange villes-campagnes et à découper le continuum rural-urbain en tranches-types. Nous avons choisi le Québec et l'Ontario comme champ d'enquête car nous y voyons deux soussystèmes d'un ensemble commun. La période 1871-1911, en plus de comprendre les années où l'on a généralement situé l'amorce de l'urbanisation, nous permet d'utiliser les données démographiques et économiques publiées dans les recensements sans trop de problèmes d'uniformisation.

D'autres données publiées plus fréquemment que les recensements nous ont aussi servi de point de départ. C'est le cas des données sur la circulation du courrier et des mandats-poste, que nous utilisons à la fois comme indicateur d'un ordre de grandeur démo- 
graphique et comme mesure de participation aux réseaux socioéconomiques. Les résultats préliminaires de la compilation de ces données sont assez encourageants. Ils démontrent une consolidation et une croissance en flèche aux paliers supérieurs du monde urbain. C'est un phénomène déjà connu, mais dont nous pouvons mieux mesurer l'ampleur. La structure du réseau urbain est donc assez bien dessinée dès 1871; elle est aussi plus rigide au Québec qu'en Ontario. Les déplacements entre les différents ordres de grandeur sont assez rares; les localités qui changent ainsi de palier se situent presque toutes en Ontario, et le jeu ne se fait qu'aux paliers inférieurs. L'analyse devra rendre compte des coudoiements dans ces zones grises difficiles à éclaircir.

Un traitement rapide des données du recensement de 1871 nous porte aux mêmes constatations. Utilisant les sous-districts de recensement comme unité d'analyse, nous en avons construit une échelle selon le pourcentage des familles n'habitant pas la ferme. Même si elle est grossière, cette échelle a deux avantages: elle part de la ferme et non de la ville, qui n'est en quelque sorte qu'un des points d'arrivée du processus d'urbanisation; et elle opère un premier découpage du continuum. On distingue sept catégories de localités: 1) les cités $(95 \%$ des familles habitant ailleurs qu'à la ferme en Ontario et $99 \%$ au Québec); 2) les villes (89\% dans les deux provinces); 3 ) les localités rurales non-agricoles $(90 \%$ et $88 \%)$; 4) les villages $(86 \%$ et $79 \%)$; 5) les localités «rurales urbanisées » $(62 \%$ et $61 \%)$; 6) les localités «rurales semi-urbanisées» $(43 \%$ et $42 \%)$; 7) les localités rurales proprement dites (21\% et $23 \%)$. Dans plus du tiers des localités recensées en 1871, au moins la moitié des familles, au Québec comme en Ontario, n'habitaient pas à la ferme. Enfin, les localités rurales non-agricoles et les villages formeraient des catégories moins homogènes que les autres selon certains critères démographiques comme la taille des familles ou les taux de fertilité.

Cette dernière constatation est-elle le fruit d'une illusion statistique? Nous indique-t-elle un phénomène de transition? Une enquête approfondie apportera peut-être la réponse. Déjà cependant, les résultats préliminaires nous incitent à poursuivre la recherche et à faire le lien entre les recensements de 1871 jusqu'à 1911, pour établir ensuite une hiérarchie diachronique. Une fois cette étape franchie, nous nous attacherons à cerner et à définir, à l'aide de données additionnelles comme les indices tirés des agences de crédit et les informations disponibles dans les bottins et les atlas, les différents réseaux socio-économiques et les sous-systèmes démo- 
graphiques que nous pouvons percevoir dès maintenant. Nous espérons que cette démarche sera utile à l'histoire rurale comme à l'histoire urbaine, et que les modèles qui en résulteront susciteront de nouvelles enquêtes monographiques sur les comportements et les attitudes à différents niveaux d'urbanisation. 